\title{
Actas del Congreso internacional sobre Megalitismo
}

\section{Charles-Tanguy Le Roux}

\section{(2) OpenEdition}

12 Journals

Édition électronique

URL : http://journals.openedition.org/rao/1468

DOI : 10.4000/rao.1468

ISBN : 978-2-7535-1846-9

ISSN : $1775-3732$

\section{Éditeur}

Presses universitaires de Rennes

\section{Édition imprimée}

Date de publication : 31 décembre 2011

Pagination : 286-289

ISBN : 978-2-7535-1844-5

ISSN : 0767-709X

\section{Référence électronique}

Charles-Tanguy Le Roux, «Actas del Congreso internacional sobre Megalitismo », Revue archéologique de l'Ouest [En ligne], 28 | 2011, mis en ligne le 30 mars 2012, consulté le 04 décembre 2020. URL

http://journals.openedition.org/rao/1468 ; DOI : https://doi.org/10.4000/rao.1468

Ce document a été généré automatiquement le 4 décembre 2020.

Tous droits réservés 


\title{
Actas del Congreso internacional sobre Megalitismo
}

\author{
Charles-Tanguy Le Roux
}

\section{RÉFÉRENCE}

Eraso, J. F. et Mujika Alestiza, J. A. (ed.), 2010 - Actas del Congreso internacional sobre

Megalitismo y otras manifestaciones funerarias contemporaneas en su contexto social, economico y cultural (Proceedings of the International Congress on the study of Megaliths and other contemporary burials in a social, economic and cultural context), Munibe, suplemento 32, 712 p. (ISBN 978-84-937670-7-5).

1 Issu de la manifestation qui s'est tenue en juin 2007 à Ordizia (province de Guipuzcoa), cet imposant volume est distribué par la Sociedad de Ciencias, Zorroagagaina 11, 20014 DONOSTIA (San Sebastian) [dazkaritza@arranzadi-zientiak.org]. Le corps de l'ouvrage comprend 9 "ponencias» (rapports synthétiques régionaux) et 28 "comunicaciones», aux sujets fort variés.

2 Les synthèses régionales ont été traitées assez librement par les auteurs qui, comme il était logique, se sont davantage appuyés sur la réalité du territoire qu'ils présentaient et sur leur sensiblité propre que sur le cannevas-type qui avait pu leur être suggéré. Il en résulte une grande variété d'approches, mais qui a su garder une certaine cohérence d'une contribution à l'autre. Un bémol toutefois: la présentation des dates radiocarbone. D'un papier à l'autre (et même à l'intérieur de certains), on se perd entre dates $\mathrm{aC}, \mathrm{AC}, \mathrm{BC}$, cal.BC et $\mathrm{BP}$, données avec ou sans fourchettes, avec (ou le plus souvent sans) tableaux de concordance. Cela ne facilite pas la compréhension ni les comparaisons interrégionales (qui étaient, peut-on présumer, le but initial). Autre problème : les contributeurs traitent de LEUR région, qu'ils connaissent par coeur et, de ce fait, peinent parfois à tenir un langage accessible aux «non-initiés» de leur microcosme scientifique (à commencer par des noms de sites ou de terroirs pas toujours faciles à localiser sur une carte générale). 
3 Une seule de ces synthèses concerne pleinement un domaine extra-hispanique; c'est celle de Luc Laporte sur l'ouest de la France (au sens large, soit pratiquement entre Seine et Dordogne, même si le « cœur de sujet » reste la Bretagne et le Centre-Ouest). Ce travail est chronologiquement centré sur la deuxième moitié du $\mathrm{V}^{\mathrm{e}}$ et le début du $\mathrm{IV}^{\mathrm{e}}$ millénaires avant J.-C., mais il est vrai que la matière y est particulièrement riche. L'auteur met l'accent sur un ensemble de phénomènes au coeur de ses préoccupations actuelles, à savoir les dynamiques de construction/destruction, de fonctionnement, de remplois et de remaniements architecturaux, qu'il concernent les chambres ou les cairns, qu'ils soient délibérés ou qu'ils résultent de réparation(s) voire d'accident(s) de chantier. À la lumière de fouilles récentes (dont Prissé-la-Charrière, Locmariaquer ou Le Souc'h entre bien d'autres), la relecture de monuments anciennement fouillés (comme les grands « tumulus carnacéens » il y a plus d'un siècle - ou même Barnenez et Carn voici un demi-siècle) s'avère particulièrement riche. En conclusion, L. Laporte propose quelques "grandes tendances» pour la période considérée et brosse un tableau bien plus complexe que les modèles jusqu'ici proposés, notamment en ce qui concerne les rapports entre monuments et espaces funéraires et tout en rappelant les pistes récemment ouvertes quant à l'héritage des différents chasseurs-cueilleurs, aux grands courants de néolithisation et aux rapports entre bâtis des vivants et demeures des morts.

4 La «submeseta norte» (entendons la Vieille-Castille de la région de Burgos à celle de Salamanque, dans l'actuelle province de Castilla y Leon) est présentée par G. Delibes de Castro. L'auteur relève tout d'abord un lien apparent entre l'implantation des mégalithes et les actuelles zones de stress hydrique estival modéré, favorables au pastoralisme (pour autant que ces données climatiques soient transposables au Néolithique...). Le polymorphisme des tombes l'amène à envisager un lien avec une éventuelle variété des usages. Successivement. Il distingue ainsi les sépultures "canoniques" (y compris celles tout ou partie en pierre sèche et pour lesquelles il rappelle l'évolution typologique naguère proposée par $M$. Rojo Guerra), les «redondiles» (chambres aux élévations possiblement en terre) et les tombes « à chape de chaux » (pour lesquelles les expérimentations tentées jusqu'ici restent non décisives quant à leur interprétation comme résultant de l'incendie, en fin d'utilisation, de chambres de type «tholos»). Cela conduit l'auteur à discuter le fonctionnement de ces différentes tombes (inhumation collective directe versus phase d'exposition des restes, transitoire ou prolongée). Une révision chronologique le conduit à discuter les phases d'implantation et de développement, le rythme des dépôts funèbres, l'ensemble de la séquence pouvant se placer entre 4200 et 3500 av. J-C sur la base des quelque 45 dates radiocarbone disponibles. L'étude des mobiliers souligne, entre bien d'autres points, la quasi-absence de céramique en phase initiale, l'ethnicité de certains objets (comme les spatules en os) et l'existence d'échanges à grande distance (haches polies allogènes, coquillages méditerranéens). quant aux perdurations, elles vont d'un important "revival » campaniforme à des réinterprétations de ce mégalithisme jusqu'en plein âge du Bronze. L'article se termine par une évocation des politiques régionales (avec leurs forces et leurs faiblesses) face aux agressions du monde moderne à l'égard de ce patrimoine.

Pour la Galice, A. Rodriguez Casal retrace l'évolution historique des concepts et de vla recherche de terrain depuis le renouveau scientifique intervenu - largement de son fait - durant les dernières décades $\mathrm{du} \mathrm{xx}^{\mathrm{e}}$ siècle. Il rappelle aussi cette spécificité 
régionale qu'est l'explosion de la perception patrimoniale du mégalithisme, à travers notamment des ensembles emblématiques comme Parxubeira et surtout Dombate (monument-phare dont les grandioses projets de mise en valeur semblent bien, hélas, avoir été des cache-misère sinon des alibis - cf. la fig. 21 clôturant l'article). L'auteur propose une périodisation ternaire du mégalithisme régional: mégalithisme initial (avec fosses individuelles et dolmens simples) de -4000 à -3500 ; plein mégalithisme (dolmens à couloir) de -3500 à -2500 ; mégalithisme final (cistes et fosses) de -2500 à -2000 , ceci avant des continuations chalcolithiques. Mais il sait aussi s'écarter de la « froideur des données » pour nous rappeler que l'art pictural des mégalithes galiciens a été reconnu dès 1874 et pour nous emmener vers la pensée symbolique et les rituels funéraires tels qu'on peut tenter de les restituer à travers les différents aspects de l'expression artistique (peintures et gravures pariétales, stèles anthropomorphes, objets à forte charge emblématique) attestés à l'intérieur des tombes mégalithiques galiciennes elles-mêmes ou à l'extérieur de celles-ci.

Quant à la contribution de M. A. de Blas Cortina sur les Asturies, elle s'appuie sur le site emblématique de Peña $\mathrm{Tu}$, cet extraordinaire rocher naturel qui domine les gorges du rio Puron à l'extrémité ouest de la Sierra plana de La Borbolla, un étroit haut-plateau intercalé entre la mer et les montagnes de la Sierra de Cuera. L'auteur souligne d'abord comment le zoomorphisme fantastique de cette roche a dû frapper les hommes depuis le Paléolithique supérieur au moins Mais le coeur de sa contribution reste la présentation des tumulus de la côte orientale des Asturies qui, pour l'essentiel (une trentaine de monuments), se rassemblent sur le plateau de La Borbolla, de quelques hectomètres à quelques kilomètres à l'est de Peña Tu. A partir des comptes-rendus d'explorations anciennes, l'auteur a pu proposer un "portrait-type» de ces monuments à ciste centrale et péristallithe. Ce portrait ne se retrouve d'ailleurs que fort imparfaitement dans le seul monument qui ait été fouillé récemment et qui n'a donné qu'un fort maigre mobilier mais une date radiocarbone dans le dernier quart du Ve millénaire av. J.-C. Puis M. A. de Blas revient au rocher lui-même pour détailler les différentes interventions humaines aujourd'hui décelables à sa base. Outre une relecture partielle du fameux « anthropomorphe et poignard " gravé et peint de la face est, il nous rappelle la présence de peintures schématiques vraisemblablement bien plus anciennes sur la face nord, associées à une petite plate-forme artificielle jusqu'ici passée inaperçue. En conclusion, la roche de Peña Tu lui apparaît comme un possible umbilicus mundi pour les populations de la région à la recherche d'un espace funéraire fédérateur.

7 Le haut bassin du Tage (de l'est de Madrid à la frontière portugaise) est présenté par $\mathrm{P}$. Bueno Ramirez, R. Barroso Bermejo et R. de Balbin Behrmann. L'émergence d'une véritable problématique sur le mégalithisme de cette région est là aussi récente (dernier quart du $\mathrm{xx}^{\mathrm{e}}$ siècle) et très largement à mettre au crédit de l'équipe qui pilote cette contribution. Les fruits de cet intense travail sont présentés de façon très dense ; plutôt qu'un "résumé de résumé » nécessairement caricatural, essayons donc d'en mettre en exergue quelques points marquants. Après un rappel du contexte géographique d'implantation des mégalithes (et avant de rappeler les potentialités aurifères et cuprifères du bassin), les auteurs réaffirment leur vision du mégalithisme comme un «système transversal » permettant, par la mise en oeuvre de méthodes très variées, l'analyse des sociétés concernées, aux plans économique (par des études taphonomiques poussées) comme socio-culturel (par le «décodage» de gestes particuliers - dépôts mobiliers, aménagements d'espaces, remplois, art, «menhirs 
indicateurs ", condamnations de chambres...). Vient ensuite une présentation «topotypo-chronologique » d'un mégalithisme particulièrement varié dans la vaste région considérée. Les études ostéologiques poussées qui ont été réalisées sont rappelées, ainsi que les 28 dates radiocarbone sur restes humains désormais disponibles, avant une conclusion (hélas un peu succincte) rappelant les prolongements, dans cette région aussi, du «mégalithisme culturel» dans le monde campaniforme puis de l'âge du Bronze.

8 La Catalogne fait l'objet de deux rapports (qui auraient peut-être mérité une meilleure coordination): M. Molist et X. Clop évoquent les origines du mégalithisme de cette région après que J. Tarrus i Galter en ait présenté le "plein mégalithisme » des $\mathrm{IV}^{\mathrm{e}}$ et III ${ }^{\mathrm{e}}$ millénaires av. J.-C.

9 Le premier travail cité rappelle les propositions classiques relatives au mégalithisme catalan avant de proposer une brève synthèse des fouilles récentes dans une région où l'Epicardial est attesté dès -5400 alors que le plein Néolithique moyen s'y développe entre -4000 et -3300 , avec son économie agro-pastorale bien consolidée et ses fameux Sepulcros de Fosa. Trois groupes de sépultures antérieures à ces derniers sont passés en revue : les tombes de l'embouchure de l'Ebre (relevant elles-mêmes de trois types bien différenciés), les nécropoles à tombes individuelles de la plaine littorale et - surtout les tumulus à ciste et couronne pierreuse du " groupe de Tavertet » découverts dans les années 1980 sur les hauteurs de l'arrière-pays, que l'on a pu paralléliser avec les débuts du groupe de Montbolo et qui témoignent désormais d'un véritable monumentalisme funéraire prémégalithique dans la région. Un rapide « état de la question » est ensuite proposé pour les Sepulcros de Fosa, désormais calés entre la fin du V $\mathrm{V}^{\mathrm{e}}$ millénaire avant J.C. et le milieu du IV où ils interfèrent avec les premiers dolmens à couloir.

10 Auparavant, J. Tarus i Galter nous avait brossé un cadre général de la Catalogne et des six «zones mégalithiques» (réparties en trois grandes aires) que l'on y reconnaît aujourd'hui, après qu'un effort de prospection ait plus que doublé le corpus depuis les années 1950 (plus de 450 monuments attestés aujourd'hui). Une typologie architecturale des tombes à couloir en quatre grands groupes («qui en outre s'échelonnent dans le temps») est proposée : à chambre subcirculaire ou polygonale puis trapézoïdale au fil du IV millénaire, avant une évolution, dès le début du III ${ }^{e}$, vers des chambres rectangulaires (avec dalles juxtaposées et non plus chevauchantes entre la chambre et le couloir). Ce dernier est désormais plus haut et plus large tandis que la structure tumulaire devient plus élaborée. Quant aux chambres simples et aux cistes, elles semblent plus tardives (mi-III ${ }^{\mathrm{e}}$ à mi-II ${ }^{\mathrm{e}}$ millénaires av. J.-C.), avec trois sous-types selon leur mode d'accès. La connaissance des rites funéraires s'est améliorée grâce à une série de fouilles récentes mais la documentation reste faible pour le type ancien (même si la condamnation du couloir y semble assez courante et le recrutement limité). L'effectif inhumé augmente sensiblement dans les tombes à couloir les plus évoluées (les galerias catalanas), en même temps que s'y notent des pratiques funéraires complexes. Quand aux cistes, hypogées et "paradolmens", ils semblent avoir largement servi d'ossuaires après décarnisation. L'auteur a également repris la question des orientations, maintes fois agitée depuis le XIX ${ }^{e}$ siècle ; Il est arrivé à des résultats complexes et contrastés qui ont été développés récemment par J. Oriol Font (2007), notamment dans le cadre d'études élargies au pourtour méditerranéen du nordouest (Provence et Languedoc). L'art « mégalithique » catalan, fort modeste et au moins pour partie lié à l'art schématique ibérique, a longtemps été négligé; révisions et 
découvertes récentes (sur des mégalithes - tombes et menhirs - mais aussi sur des rochers naturels) le montrent cependant plus varié qu'il n'y paraissait. Là aussi, des rapprochements ont été proposés avec la Provence, la Suisse, les grands Causses et même la façade atlantique. Enfin, l'article se termine par une présentation des habitats connus en relation avec le mégalithisme.

11 Le Pays basque fait également l'objet de deux contributions. Les formes funéraires des «Pyrénées occidentales» (en fait, l'actuelle Navarre) sont présentées par M. A. Berguiristain Gurpide, J. Sesma Sesma et J. Garcia Gazolaz. Un «catalogue des évidences" nous présente successivement les données récentes quant aux "manifestations funéraires prémégalithiques", puis sur le "collectivisme funéraire mégalithique " (avec huit sites récemment documentés qui, tous sauf un, se trouvent hors de la zone "dolménique classique» des montagnes du nord de la province); dommage que l'ensemble des relevés - apparemment fort précis - de la chambre d'Aizibita (fig. 3) aient été réduits à un quart de page, ce qui les rend quasi-illisibles (même avec une loupe!). Les "autres formes funéraires collectives contemporaines " (3 sites présentés) précèdent les diverses «manifestations post-mégalithiques » antérieures à la généralisation de l'incinération (12 sites). De copieuses conclusions reprennent ces données de manière transversale; sont ainsi mis en exergue l'hétérogénéité des architectures (avec divers systèmes de fermeture et «espaces dédiés » aménagés dans les chambres), les études taphonomiques et paléopathologiques débouchant sur des considérations sociétales (violence, démographie), ainsi que les quelque 50 datations radiocarbone (malheureusement illustrées par une fig. 10 inutilisable et sans le tableau synthétique qui aurait été bienvenu), pour déboucher sur la problématique régionale du passage vers l'âge du Bronze.

J. Fernadez Eraso, A. Mujica Alustiza et X. Penalver Iribarren présentent ensuite l'habitat et le monde funéraire du Pays basque (pris dans sa totalité cette fois), au long de la préhistoire récente. Cela va des premières installations néolithiques $d u-V I^{e}$ millénaire (à partir du monde Cardial via le bassin de l'Ebre - cf. la spectaculaire stratigraphie de Los Husos II donnée en fig. 1) jusqu'aux utilisations tardives de l'âge du Fer voire de l'Antiquité. Une chrono-typologie des sépultures mégalithiques est esquissée, depuis les sepulcros de corredor (hélas chiches en dates utilisables) jusqu'aux "dolmens simples ", mieux documentés et qui représentent en fait $90 \%$ du corpus. Ces derniers semblent apparaître vers $5300 \mathrm{BP}$ avec une construction se poursuivant jusqu'au milieu du III ${ }^{\mathrm{e}}$ millénaire et des réutilisations nombreuses jusqu'en plein âge du Bronze voire bien plus tard (d'où la large fourchette chronologique annoncée au départ). Le lien avec les habitats permet à l'auteur d'aborder le problème des "cromlec'hs» ou circulos pirenaicos (1 452 connus, regroupés en 558 ensembles). A cheval sur les Monts cantabres et les Pyrénées, le Pays basque est aussi tourné vers la Méditerranée via le bassin de l'Ebre (cf. supra). Dans cette partie sud, se développent aussi d'autres modes funéraires (champs d'urnes et groupes de cistes), dont la datation semble encore précaire. Mais dommage que cette synthèse pèche globalement par une présentation chronologique bien confuse (passages incessants du «cal.» au «BP », écarts-types disparates dans les tableaux, voire lapsus dans la nomenclature des millénaires...).

13 Il est hélas matériellement impossible de rendre compte de manière détaillée des 28 autres articles correspondant aux comunicaciones, et c'est bien dommage tant leur 
intérêt et leur diversité sont grands. On y trouve à la fois des contributions monographiques et des synthèses méthodologiques. Les unes comme les autres relèvent d'approches très diverses et parfois fort pointues, témoignant de la vitalité de l'école espagnole de préhistoire. Quelques thèmes sont manifestement privilégiés, dont au premier chef l'archéologie du paysage sous ses différentes facettes : marqueurs de territoire (J. Agirre Garcia et al....), paléoenvironnement végétal (M. J. Iriarte ; M. Ruiz Alonso et al.), organisation de l'espace à l'échelle d'une micro-région (J. A. Alfonso Marrero et al.) ou d'un plus vaste pays (J. M. Edeso et al. ; J. A. Camara Serrano et al. ; F. Galilea Martinez; J. A. Lopez Saez et al.). Le lien est donc tout trouvé avec les études par SIG (L. Ernetta Altarriba et N. Cormo) ou les prospections-inventaires qui nous sont présentées (L. Milan San Emeterio ; M. A Moreno Gallo ; S. San José), ces dernières étant naturellement relayées par deux présentations de politiques de mise en valeur, l'une et l'autre au Pays basque, avec leurs succès et leurs déboires comme partout (E. Alvarez Vidaurre; M. Ceberio Rodriguez). L'anthropologie physique est également à l'honneur, notamment avec des travaux de paléo-génétique (S. Cardoso; M. Hervella et al.). Les pratiques funéraires sont abordées à plusieurs reprises, soit directement (T. Fernandez Crespo; J. C. Lopez Quintana et al. ; X. Clop; J. Vega et al.), soit à travers l'étude de certains types de mobiliers : céramique (J. F. Gibaja et al.), métal (M. J. Sanchez Sierra ; J. A. Mujika Alustiza), parures (J. I. Vegas Arramburu), grottes funéraires (O. Sarobe Escudero). Malgré le qualificatif « international » donné à ce colloque, les contributions extra-espagnoles y restent peu abondantes: outre celle de L. Laporte (cf. supra), on trouve la synthèse de $\mathrm{M}$. Sohn sur les dépôts "collectifs" dans le nord-ouest de l'Europe, celle de L. Spanedda et al. sur le golfe d'Orosei en Sardaigne, plus - à la limite les comparaisons avec le Rouergue proposées par P. Martinez et al. pour la statuemenhir catalane de Ca l'Estrada.

Tous les articles sont en espagnol, sauf ceux de L. Laporte et de M. Sohn (en français) et ceux de J. A. Mujika et al. et de S. San José (en basque). Tous comportent mots-clés et résumés en espagnol, basque, anglais et parfois français (mais les légendes des figures, parfois succinctes (voire absentes), ne sont pas toujours traduites, ce qui pose problème pour les articles en basque notamment). Globalement, la présentation est soignée, avec quadrichromie quasi-intégrale (mais quelques problèmes de mise en page et de qualité graphique demeurent ici et là). Au total, et malgré l'absence d'un fil directeur clair dans l'ordre des articles, cet important volume est une mine d'informations sur toute une branche de cette archéologie espagnole que l'on sait en pleine fermentation. 